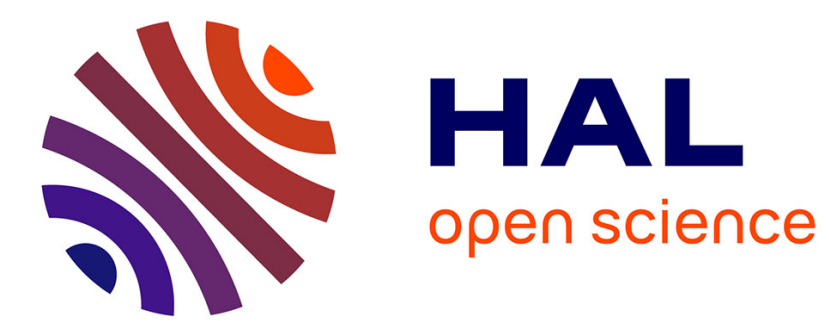

\title{
COMPUTING QUOTIENTS BY CONNECTED SOLVABLE GROUPS
}

\author{
Gregor Kemper
}

\section{To cite this version:}

Gregor Kemper. COMPUTING QUOTIENTS BY CONNECTED SOLVABLE GROUPS. MEGA 2019 - International Conference on Effective Methods in Algebraic Geometry, Jun 2019, Madrid, Spain. hal-02912346

\author{
HAL Id: hal-02912346 \\ https://hal.inria.fr/hal-02912346
}

Submitted on 5 Aug 2020

HAL is a multi-disciplinary open access archive for the deposit and dissemination of scientific research documents, whether they are published or not. The documents may come from teaching and research institutions in France or abroad, or from public or private research centers.
L'archive ouverte pluridisciplinaire HAL, est destinée au dépôt et à la diffusion de documents scientifiques de niveau recherche, publiés ou non, émanant des établissements d'enseignement et de recherche français ou étrangers, des laboratoires publics ou privés. 


\title{
COMPUTING QUOTIENTS BY CONNECTED SOLVABLE GROUPS
}

\author{
GREGOR KEMPER
}

\begin{abstract}
Consider an action of a connected solvable group $G$ on an affine variety $X$. This paper presents an algorithm that constructs a semi-invariant $f \in K[X]=: R$ and computes the invariant ring $\left(R_{f}\right)^{G}$ together with a presentation. The morphism $X_{f} \rightarrow \operatorname{Spec}\left(\left(R_{f}\right)^{G}\right)$ obtained from the algorithm is a universal geometric quotient. In fact, it is even better than that: a so-called excellent quotient. If $R$ is a polynomial ring, the algorithm requires no Gröbner basis computations. If $R$ is a complete intersection, then so is $\left(R_{f}\right)^{G}$.
\end{abstract}

\section{INTRODUCTION}

In the theory of algebraic groups, two cases stand out as being well understood: reductive groups and solvable groups. While the invariant theory of reductive groups is well-behaved and, in many aspects, well understood, this is not the case for solvable and, in particular, unipotent groups. For example, invariant rings of unipotent groups need not be finitely generated, and even if they are, categorical quotients need not exist (see Ferrer Santos and Rittatore [8, Example 4.10]). Notice that if $G$ is a connected linear algebraic group acting on an affine variety $X$ and $B$ is a Borel subgroup, then $K[X]^{G}=K[X]^{B}$ (see Humphreys [15, Exercise 21.8]); so computing invariant rings of connected solvable groups would mean computing invariant rings of all connected groups. This goal is still out of reach, but it makes the invariants of connected solvable groups particularly interesting.

There is a sizeable list of papers devoted to the invariant theory of the additive group (e.g. Tan [23], van den Essen [5], Freudenburg [9], Derksen and Kemper [3, Section 3.1], and Tanimoto [24]), unipotent groups (e.g. Hochschild and Mostow [14], Grosshans [13], Fauntleroy [6, 7], Bérczi et al. [1], Greuel and Pfister [11, 12], and Sancho de Salas [22]), and connected solvable groups (Rosenlicht [19, Section 4] and Popov [18]). Most relevant in our context is the recent paper [18], in which it is shown that if a connected solvable group $G$ acts on an irreducible variety $X$ over an algebraically closed field $K$, then $X$ has a $G$-stable dense open subset $U \subseteq X$ that admits a geometric quotient $U \rightarrow Y$ such that, in addition, there is an isomorphism $U \stackrel{\sim}{\longrightarrow} \mathbb{A}^{r, s} \times Y$, with $\mathbb{A}^{r, s}=\left\{\left(\xi_{1}, \ldots, \xi_{r+s}\right) \in \mathbb{A}^{r+s} \mid \xi_{1} \cdots \xi_{s} \neq 0\right\}$, such that the diagram

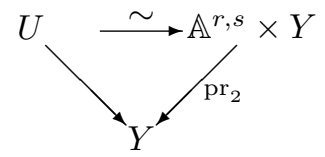

(with $\mathrm{pr}_{2}$ the second projection) commutes. This result is nonconstructive, mainly since Rosenlicht's general result [21] about geometric quotients on suitable open subsets is used for obtaining a geometric quotient.

The main goal of this paper is to make Popov's result constructive, and to show that the computations required for this are exceptionally easy. Under the assumption that $G$ is a connected solvable group acting on an irreducible affine variety $X$, our algorithm constructs a suitable nonzero semi-invariant $f$ in the coordinate ring $R:=K[X]$ and computes the invariant ring $\left(R_{f}\right)^{G}$ together with a presentation, such that the induced map from $U=X_{f}:=\{x \in X \mid f(x) \neq 0\}$ to $Y:=\operatorname{Spec}\left(\left(R_{f}\right)^{G}\right)$ satisfies Popov's result. All that is required for the algorithm are arithmetic operations and zero recognition in $R$. So for example if $X=\mathbb{A}^{n}$, the algorithm does not need any

2010 Mathematics Subject Classification. 14L24, 13A50, 14L30.

Key words and phrases. Algorithmic invariant theory, solvable groups, geometric invariant theory, geometric quotient. 
GREGOR KEMPER

Gröbner basis computations. It turns out that $U \rightarrow Y$ is even a universal geometric quotient. The relations between the generators of $\left(R_{f}\right)^{G}$ computed by the algorithm reveal that $\left(R_{f}\right)^{G}$ is a complete intersection if $R$ is one, for example in the case $X=\mathbb{A}^{n}$. This result seems to be new.

The paper starts by studying quotients of a type modeled after the above-mentioned result by Popov [18] (and also the one by Greuel and Pfister [11] on unipotent group actions), which we propose to call excellent quotients. Since connected solvable groups are built from copies of additive and multiplicative groups, Sections 2 and 3 treat actions of these groups. The results lead to algorithms for computing excellent quotients, which are then put together in the final section to obtain an algorithm for a connected solvable group. A difficulty with this iterative approach is that when computing the invariant ring $\left(R_{f}\right)^{H}$ of a normal subgroup $H \subseteq G$, the element $f$ must be chosen as a semi-invariant of $G$, not just of $H$, since otherwise $G$ does not act on $\left(R_{f}\right)^{H}$.

An extended preprint version of this article has appeared in the arXiv [16]. In that version, $K$ need not be an algebraically closed field but can be any ring. The irreducibility hypothesis on $X$ is also dropped: it can be any affine $K$-scheme. The preprint [16] is more than twice as long as this paper, and there is no intention of publishing it anywhere else than in the arXiv.

Acknowledgements. This article benefited from interesting and helpful conversations with Igor Dolgachev, Hanspeter Kraft, Stephan Neupert, and Vladimir Popov. I also thank the anonymous referees for their helpful comments.

\section{Geometric overture: EXCEllent Quotients}

In the following, all varieties and algebraic groups are assumed to be over an algebraically closed field $K$. Let $G$ be an algebraic group acting morphically on a variety $X$. We say that a morphism $X \rightarrow Y$ to another variety is an excellent quotient if

(i) $X \rightarrow Y$ is a universal geometric quotient (see Mumford et al. [17, Definitions 0.6 and 0.7$]$ ) and

(ii) There is an isomorphism $X \stackrel{\sim}{\longrightarrow} F \times Y$, with $F$ another variety, such that the diagram

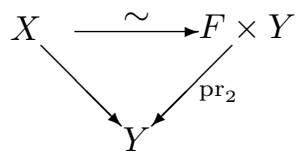

(with $\mathrm{pr}_{2}$ the second projection) commutes.

If we wish to be more specific, we will call $X \rightarrow Y$ an excellent quotient by $G$ with fibers $F$.

It follows immediately that all $G$-orbits in $X$ are isomorphic to $F$. By picking a point from $F$ we obtain a morphism $Y \rightarrow F \times Y$, and composing this with $F \times Y \stackrel{\sim}{\longrightarrow} X$ provides a morphism $Y \rightarrow X$, which we call a cross section since the composition $Y \rightarrow X \rightarrow Y$ is the identity.

An excellent quotient can be defined for schemes (over an arbitrary ground scheme $S$ instead of an algebraically closed field). In this case the existence of an $S$-valued point of $F$ is required for obtaining a cross section. Everything in this section carries over to the scheme-theoretic setting (see [16]).

Recall that the definition of a geometric quotient in [17] has four parts and is a bit cumbersome. But as the following result shows, the presence of a cross section makes it much easier to check whether the quotient is (universally) geometric, especially if $X$ and $Y$ are affine.

Proposition 1.1. Let $G$ be an algebraic group acting on an affine variety $X$ and let $X \rightarrow Y$ be a morphism to an affine variety $Y$ with a cross section $Y \rightarrow X$. Then $X \rightarrow Y$ is a universal geometric quotient if and only if

(a) the composition

$$
G \times Y \rightarrow G \times X \stackrel{\text { act }}{\longrightarrow} X
$$

(with the last map given by the $G$-action) is surjective, and 
(b) for every homomorphism $K[Y] \rightarrow A$ of rings we have

$$
\left(A \otimes_{K[Y]} K[X]\right)^{G}=A
$$

(which implies $K[X]^{G}=K[Y]$ ).

Since the image of the cross section meets every fiber of $X \rightarrow Y$ in precisely one point, (a) says that the fibers of $X \rightarrow Y$ are precisely the $G$-orbits.

Proof. Let us first assume that (a) and (b) hold. Then $K[X]^{G}=K[Y]$ implies that the diagram

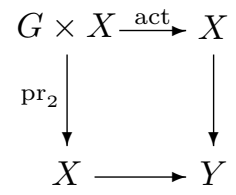

commutes. Let $Y^{\prime} \rightarrow Y$ be a morphism of schemes. We need to show that the map $X^{\prime}:=$ $Y^{\prime} \times_{Y} X \rightarrow Y^{\prime}$ obtained by base change is a geometric quotient. The above diagram remains commutative after replacing $Y$ by $Y^{\prime}$ and $X$ by $X^{\prime}$. Moreover, the morphism $X^{\prime} \rightarrow Y^{\prime}$ also has a cross section, which implies that it is surjective and submersive. (Recall that submersive means that a subset of $Y^{\prime}$ is open if its preimage in $X^{\prime}$ is open). We need to show that the morphism $G \times X^{\prime} \rightarrow X^{\prime} \times_{Y^{\prime}} X^{\prime}$ given by applying the $G$-action and the second projection is surjective. Since surjectivity can be shown by considering points with values in large enough fields (see Görtz and Wedhorn [10, Proposition 4.8]), this comes down to proving that the fibers of $X^{\prime} \rightarrow Y^{\prime}$ are precisely the $G$-orbits. But this is precisely what the surjectivity of $G \times Y^{\prime} \rightarrow G \times X^{\prime} \rightarrow X^{\prime}$, which follows from (a) (see [10, Proposition 4.32]), says.

Finally, we have to show that for an open subset $V \subseteq Y^{\prime}$ with preimage $U \subseteq X^{\prime}$, the map $\Gamma\left(V, \mathcal{O}_{Y^{\prime}}\right) \rightarrow \Gamma\left(U, \mathcal{O}_{X^{\prime}}\right)$ induced by $X^{\prime} \rightarrow Y^{\prime}$ has $\Gamma\left(U, \mathcal{O}_{X^{\prime}}\right)^{G}$ as its image. The cross section gives a left inverse to the map $\mathcal{O}_{Y^{\prime}} \rightarrow \mathcal{O}_{X^{\prime}}$ of sheaves, so $\Gamma\left(V, \mathcal{O}_{Y^{\prime}}\right) \rightarrow \Gamma\left(U, \mathcal{O}_{X^{\prime}}\right)$ is injective. This means that an $f \in \Gamma\left(U, \mathcal{O}_{X^{\prime}}\right)$ has at most one inverse image in $\Gamma\left(V, \mathcal{O}_{Y^{\prime}}\right)$, and such an inverse image can be glued together from inverse images of restrictions of $f$ to preimages of affine open subsets of $V$. We may therefore assume $V$ to be affine, say $V=\operatorname{Spec}(A)$. Since $U=V \times_{Y} X$, this implies $U=\operatorname{Spec}\left(A \otimes_{K[Y]} K[X]\right)$. The map $X^{\prime}=Y^{\prime} \times_{Y} X \rightarrow Y^{\prime}$ is just the first projection, so $U \rightarrow V$ is also the first projection, and it follows that $A=\Gamma\left(V, \mathcal{O}_{Y^{\prime}}\right) \rightarrow$ $\Gamma\left(U, \mathcal{O}_{X^{\prime}}\right)=A \otimes_{K[Y]} K[X]$ maps an $a \in A$ to $a \otimes 1$. Hence the image is $A \otimes 1$, which by (b) is equal to $\left(A \otimes_{K[Y]} K[X]\right)^{G}=\Gamma\left(U, \mathcal{O}_{X^{\prime}}\right)^{G}$. This completes the proof that $X \rightarrow Y$ is a universal geometric quotient.

Conversely, if $X \rightarrow Y$ is a universal geometric quotient, then (a) follows since the fibers are the orbits, and (b) is true since, as we have seen, it says that for all affine schemes $Y^{\prime}$ with morphisms $Y^{\prime} \rightarrow Y$, the map $\Gamma\left(Y^{\prime}, \mathcal{O}_{Y^{\prime}}\right) \rightarrow \Gamma\left(X^{\prime}, \mathcal{O}_{X^{\prime}}\right)$ has $\Gamma\left(X^{\prime}, \mathcal{O}_{X^{\prime}}\right)^{G}$ as its image.

We will deal with solvable groups by iterating excellent quotients along a chain of subgroups. This is possible thanks to the following result.

Theorem 1.2. Let $G$ be an algebraic group acting morphically on an affine variety $X$. Let quo $_{1}: X \rightarrow Y$ be an excellent quotient by a closed normal subgroup $H \subseteq G$ with fibers $F_{1}$, with $Y$ affine. Since $G$ acts on $K[X]^{H}=K[Y]$, it also acts on $Y$, with $H$ in the kernel of the action. Assume that $Y$ admits an excellent quotient quo $_{2}: Y \rightarrow Z$ by $G$ with fibers $F_{2}$, again with $Z$ affine. Then the composition $\mathrm{quo}_{2} \circ \mathrm{quo}_{1}: X \rightarrow Z$ is an excellent quotient by $G$ with fibers $F_{1} \times F_{2}$.

Remark. The theorem also holds without assuming that $X, Y$ or $Z$ are affine (see [16]). But we only need the affine case here, whose proof is less involved. 


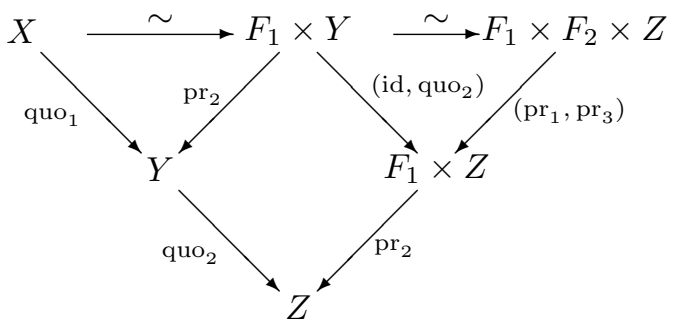

shows that the second property (ii) of an excellent quotient is satisfied. Composing cross sections sect $_{1}: Y \rightarrow X$ and sect $_{2}: Z \rightarrow Y$ gives a cross section sect $:=$ sect $_{1} \circ$ sect $_{2}$ of $X \rightarrow Z$. Since (a) and (b) of Proposition 1.1 hold for the quotients $\mathrm{quo}_{1}$ and $\mathrm{quo}_{2}$, we have to show that they also hold for the composition.

To prove (a), let $x \in X$ and set $y:=\mathrm{quo}_{1}(x), z:=\mathrm{quo}_{2}(y)$. We must show that $x$ and $x^{\prime}:=$ $\operatorname{sect}(z)$ lie in the same $G$-orbit. There exists $g \in G$ such that $y=g\left(\operatorname{sect}_{2}(z)\right)$. Since $q^{2} o_{1}$ is $G$-equivariant, we obtain

$$
\mathrm{quo}_{1}\left(g\left(x^{\prime}\right)\right)=g\left(\mathrm{quo}_{1}\left(\operatorname{sect}_{1}\left(\operatorname{sect}_{2}(z)\right)\right)\right)=g\left(\operatorname{sect}_{2}(z)\right)=y=\mathrm{quo}_{1}(x),
$$

so $g\left(x^{\prime}\right)$ and $x$ lie in the same $H$-orbit, and we are done.

To prove (b), let $K[Z] \rightarrow A$ be a ring homomorphism. Then $\left(A \otimes_{K[Z]} K[Y]\right)^{G}=A$. Moreover, applying (b) to the induced homomorphism $K[Y] \rightarrow A \otimes_{K[Z]} K[Y]$ yields

$$
\left(A \otimes_{K[Z]} K[X]\right)^{H}=\left(A \otimes_{K[Z]} K[Y] \otimes_{K[Y]} K[X]\right)^{H}=A \otimes_{K[Z]} K[Y],
$$

so $\left(A \otimes_{K[Z]} K[X]\right)^{G}=\left(A \otimes_{K[Z]} K[Y]\right)^{G}=A$.

\section{Additive group ACtions}

In this section we consider a nontrivial morphic action of the additive group $\mathbb{G}_{a}$ on an irreducible affine variety $X$. Again, we work over an algebraically closed field $K$ and remark that everything from this section carries over to the situation where $X$ is an integral affine scheme over $\operatorname{Spec}(K)$ with $K$ a ring (see [16]).

With $R:=K[X]$, the action is given by a homomorphism $\varphi: R \rightarrow R[z]$ of $K$-algebras, with $z$ an indeterminate. For $s \in R$ with $\varphi(s)=\sum_{i=0}^{d} c_{i} z^{i}$ with $c_{d} \neq 0$, we write $\operatorname{deg}(s):=d$. We have

$$
c_{0}=s \quad \text { and } \quad \operatorname{deg}\left(c_{i}\right) \leqslant d-i
$$

(see Tanimoto [24]), and in particular $c_{d} \in R^{\mathbb{G}_{a}}$ is an invariant. Following Tanimoto and various other authors, we call $s$ a local slice if it is of minimal positive degree. We call $c:=c_{d}$ the denominator of the local slice. This is because we can perform division with remainder by $\varphi(s)$ over the localization $R_{c}:=R\left[c^{-1}\right]$ : Extending $\varphi$ to $R_{c}$, for $a \in R_{c}$ we have

$$
\varphi(a)=g \cdot \varphi(s)+r
$$

with $g, r \in R_{c}[z], \operatorname{deg}_{z}(r)<d$. Crucially, it follows from [24, Lemma 2.2] that $\operatorname{deg}_{z}(g)=$ $\operatorname{deg}(g(0))$ and $\operatorname{deg}_{z}(r)=\operatorname{deg}(r(0))$, so

$$
\operatorname{deg}(g(0))<\operatorname{deg}(a), \quad \operatorname{deg}_{z}(r)=0 \quad \text { and } \quad r \in R^{\mathbb{G}_{a}}
$$

since $s$ is a local slice. Sancho de Salas [22, Section 3] and Tanimoto [24, Section 3] presented essentially identical algorithms for computing a local slice. These use division with remainder as above, and only require addition, multiplication and zero recognition in $R$; so if $R$ is, for example, a finitely generated subalgebra of a rational function field, a local slice can be computed without any Gröbner basis calculations. A variant of these algorithms that works in a more general situation and is also a bit simpler can be found in [16].

The utility of local slices can be seen from the following theorem. For example, by part (b), generators of $R_{c}^{\mathbb{G}_{a}}$ can be determined immediately if a local slice is known. Moreover, together with Proposition 1.1, parts (a), (c), and (d) imply that the map $X_{c}:=\{x \in X \mid c(x) \neq 0\} \rightarrow$ $\operatorname{Spec}\left(R_{c}^{\mathbb{G} a}\right)$ is an excellent quotient with fibers $\mathbb{A}^{1}$. While parts (a) and (b) are essentially well known, (c) and (d) seem to be new. 
Theorem 2.1. In the above situation, let $s$ be a local slice of degree $d$ with denominator $c \in R^{\mathbb{G}_{a}}$.

(a) The homomorphism $R_{c}^{\mathbb{G}_{a}}[x] \rightarrow R_{c}$ sending the indeterminate $x$ to $s$ is an isomorphism. We write $\psi: R_{c} \rightarrow R_{c}^{\mathbb{G} a}[x]$ for the inverse isomorphism.

(b) The composition

$$
\pi: R_{c} \stackrel{\psi}{\longrightarrow} R_{c}^{\mathbb{G}_{a}}[x] \stackrel{x \mapsto 0}{\longrightarrow} R_{c}^{\mathbb{G}_{a}}
$$

is a homomorphism of $R_{c}^{\mathbb{G} a}$-algebras with $\operatorname{ker}(\pi)=s R_{c}$. In particular, $\pi$ is surjective. For $a \in R_{c}, \pi(a)$ is given by

$$
\varphi(a)=g \cdot \varphi(s)+\pi(a)
$$

as in (2.2).

(c) The composition

$$
R_{c} \stackrel{\varphi}{\longrightarrow} R_{c}[z] \stackrel{\pi}{\longrightarrow} R_{c}^{\mathbb{G}_{a}}[z]
$$

(with $\pi$ applied coefficient-wise) is injective and makes $R_{c}^{\mathbb{G}_{a}}[z]$ into an $R_{c}$-module that is generated by $d$ elements. In particular, if $d=1$, then it is an isomorphism.

(d) Let $A$ be a ring with a homomorphism $R_{c}^{\mathbb{G}_{a}} \rightarrow A$. Then

$$
\left(A \otimes_{R_{c}^{\mathbb{G}} a} R\right)^{\mathbb{G}_{a}}=A
$$

Proof. (a) To show that the map is injective, let $f \in R_{c}^{\mathbb{G}_{a}}[x]$ with $f(s)=0$. Since $\varphi$ is a homomorphism of $R_{c}^{\mathbb{G}_{a}}$-algebras, this implies $f(\varphi(s))=0$, so $f=0$. To prove surjectivity, let $a \in R_{c}$. Evaluating (2.2) at $z=0$, we get $a=g(0) s+r(0)$, and (2.3) yields $r(0)=$ $r \in R_{c}^{\mathbb{G}_{a}}$. Since also $\operatorname{deg}(g(0))<\operatorname{deg}(a)$, the surjectivity follows by induction on $\operatorname{deg}(a)$.

(b) The first statement follows from (a). For the second statement, observe that the map that is claimed to be equal to $\pi$ is a homomorphism of $R_{c}^{\mathbb{G}_{a}}$-algebras, since the remainder of $\varphi(a)$ from division by $\varphi(s)$ has degree 0 by (2.3). Since $R_{c}=R_{c}^{\mathbb{G}_{a}}[s]$, it suffices to check the equality of the maps for $a=s$, which is immediate.

(c) Let $a \in R_{c}$. By (a) we may write $a=f(s)$ with $f \in R_{c}^{\mathbb{G}_{a}}[x]$. Writing $g:=\varphi(s)$ we obtain

$$
\pi(\varphi(a))=\pi(\varphi(f(s)))=f(\pi(g))=f(\pi(g-s)+\pi(s))=f(g-s)
$$

since $\pi(s)=0$ and, by (2.1), all coefficients of $g-s$ have degree $<d$ and are therefore invariants in $R_{c}^{\mathbb{G}_{a}}$. Since $g-s \in R_{c}[z]$ is nonconstant, injectivity of $\pi \circ \varphi$ follows. For $a=s$, the above equality shows that $g-s$ lies in the image of $\pi \circ \varphi$. So the polynomial $(g(x)-s)-(g-s) \in R_{c}^{\mathbb{G}_{a}}[z][x]$, which is satisfied by $z$, has coefficients (as a polynomial in $x$ ) in the image. This proves the second statement.

(d) By (a), the element $1 \otimes s \in A \otimes_{R_{c}^{\mathbb{G} a}} R:=R^{\prime}$ is algebraically independent over $A$, and $R^{\prime}=A[1 \otimes s]$. By definition, $\left(R^{\prime}\right)^{\mathbb{G}_{a}}=\operatorname{ker}\left(\varphi^{\prime}-\mathrm{id}\right)$ with $\varphi^{\prime}: R^{\prime} \rightarrow R^{\prime}[z]$ obtained by tensoring $\varphi$. Let $r \in R^{\prime}$ and write $r=\sum_{i=0}^{k} a_{k}(1 \otimes s)^{i}$ with $a_{i} \in A, a_{k} \neq 0$. With the given map $\eta: R_{c}^{\mathbb{G}_{a}} \rightarrow A$ applied to $\mathbb{R}_{c}^{\mathbb{G}_{a}}[z]$ coefficient-wise, we obtain

$$
\varphi^{\prime}(r)=\sum_{i=0}^{k} a_{i}(1 \otimes g)^{i}=\sum_{i=0}^{k} a_{i}(\eta(g-s) \otimes 1+1 \otimes s)^{i} .
$$

If $k>0$, the coefficient of $z^{k d}$ of this is $a_{k} \eta(c)^{k} \otimes 1$, which is nonzero since $\eta(c)$ is invertible in $A$. So if $r \in\left(R^{\prime}\right)^{\mathbb{G}_{a}}$, then $k=0$ and therefore $r \in A \otimes 1$, which we wrote as $A$ in the statement (d). The reverse inclusion $A \otimes 1 \subseteq\left(R^{\prime}\right)^{\mathbb{G}_{a}}$ is clear.

The aim of the following simple example is to illustrate how Theorem 2.1 can be used to compute the invariant ring $R_{c}^{\mathbb{G}_{a}}$, providing an excellent quotient. The example will be continued in Section 4.

Example 2.2. $\mathrm{SL}_{2}$-actions on binary forms are a staple of classical invariant theory. Here we consider the action of the upper unipotent subgroup $\mathbb{G}_{a} \subseteq \mathrm{SL}_{2}(\mathbb{C})$ on binary forms of degree 2 . Explicitly, $\alpha \in \mathbb{G}_{a}$ acts on $X=\mathbb{C}^{3}$ by the matrix $\left(\begin{array}{ccc}1 & \alpha & \alpha^{2} \\ 0 & 1 & 2 \alpha \\ 0 & 0 & 1\end{array}\right)$. With $R=\mathbb{C}\left[a_{1}, a_{2}, a_{3}\right]$ the trivariate polynomial ring, the action is given by the homomorphism $\varphi: \mathbb{C}\left[a_{1}, a_{2}, a_{3}\right] \rightarrow \mathbb{C}\left[a_{1}, a_{2}, a_{3}\right][z]$ with

$$
\varphi\left(a_{1}\right)=a_{1}+z a_{2}+z^{2} a_{3}, \varphi\left(a_{2}\right)=a_{2}+2 z a_{3}, \varphi\left(a_{3}\right)=a_{3} .
$$


Clearly $s=a_{2}$ is a local slice of degree 1 with denominator $c=a_{3}$. We have $g=\varphi(s)=2 a_{3} z+a_{2}$, so by the last statement from Theorem 2.1(b), the projection $\pi: \mathbb{C}\left[a_{1}, a_{2}, a_{3}^{ \pm 1}\right] \rightarrow \mathbb{C}\left[a_{1}, a_{2}, a_{3}^{ \pm 1}\right]^{\mathbb{G}_{a}}$ is given by first applying $\varphi$ and then substituting $z=\frac{-a_{2}}{2 a_{3}}$. We obtain

$$
\pi\left(a_{1}\right)=a_{1}-\frac{a_{2}}{2 a_{3}} a_{2}+\frac{a_{2}^{2}}{4 a_{3}^{2}} a_{3}=\frac{4 a_{1} a_{3}-a_{2}^{2}}{4 a_{3}}, \pi\left(a_{2}\right)=0, \pi\left(a_{3}\right)=a_{3} .
$$

Theorem 2.1 now tells us that

$$
\mathbb{C}\left[a_{1}, a_{2}, a_{3}^{ \pm 1}\right]^{\mathbb{G}_{a}}=\mathbb{C}\left[a_{2}^{2}-4 a_{1} a_{3}, a_{3}^{ \pm 1}\right]
$$

and that the morphism $X_{c}=\mathbb{C}^{2} \times \mathbb{C}^{\times} \rightarrow \mathbb{C} \times \mathbb{C}^{\times}$given by evaluating the discriminant $a_{2}^{2}-4 a_{1} a_{3}$ and $a_{3}$ is an excellent quotient with fibers $\mathbb{A}^{1}$. It is elementary to verify that the fibers of this map are indeed the $\mathbb{G}_{a}$-orbits.

In this example, an easy argument infers from (2.5) that the nonlocalized invariant ring is $\mathbb{C}\left[a_{1}, a_{2}, a_{3}\right]^{\mathbb{G}_{a}}=\mathbb{C}\left[a_{2}^{2}-4 a_{1} a_{3}, a_{3}\right]$. But the corresponding morphism $\mathbb{C}^{3} \rightarrow \mathbb{C}^{2}$ is not a geometric quotient. In fact, the fiber of a point $(\delta, 0) \in \mathbb{C}^{2}$ consists of two orbits if $\delta \neq 0$, and infinitely many if $\delta=0$.

\section{Multiplicative Group aCtions}

This section deals with an action of the multiplicative group $\mathbb{G}_{m}$ on an irreducible affine variety $X$. With $R:=K[X]$ as above, such an action is given by a homomorphism $\varphi: R \rightarrow R\left[t^{ \pm 1}\right]$ into the Laurent polynomial ring. An element $c \in R$ is called a semi-invariant of weight $k$ if $\varphi(c)=t^{k} c$. In this case $\varphi$ uniquely extends to a homomorphism $R_{c} \rightarrow R_{c}\left[t^{ \pm 1}\right]$, which will also be written as $\varphi$. For $a \in R$ we write $\operatorname{deg}(a):=\max \left\{|k| \mid t^{k}\right.$ occurs in $\left.\varphi(a)\right\}$, with $\operatorname{deg}(0):=0$. So the invariant ring $R^{\mathbb{G}_{m}}$ consists of the elements of degree 0 .

We define the notion of a local slice for the multiplicative group as follows: Let $0 \neq c \in R$ be a semi-invariant. An element $s \in R_{c}$ is called a local slice of degree $d>0$ with denominator $c$ if

(i) $s$ is a semi-invariant of weight $-d$,

(ii) $s$ is invertible in $R_{c}$, and

(iii) all elements from $R_{c} \backslash R_{c}^{\mathbb{G}_{m}}$ have degree at least $d$.

In the next section we will present an algorithm for computing a local slice which is additionally a semi-invariant with respect to a torus action. The following theorem is analogous to Theorem 2.1 and shows that $X_{c} \rightarrow \operatorname{Spec}\left(R_{c}^{\mathbb{G}_{m}}\right)$ is an excellent quotient with fibers $\mathbb{A}^{1} \backslash\{0\}$. Part (a) can also be found in Popov [18].

Theorem 3.1. In the above situation, let $s$ be a local slice of degree $d$ with denominator $c$.

(a) The homomorphism $\left(R_{c}\right)^{\mathbb{G}_{m}}\left[y^{ \pm 1}\right] \rightarrow R_{c}$ sending the indeterminate $y$ to $s$ is an isomorphism. We write $\psi: R_{c} \rightarrow\left(R_{c}\right)^{\mathbb{G}_{m}}\left[y^{ \pm 1}\right]$ for the inverse isomorphism.

(b) The composition

$$
\pi: R_{c} \stackrel{\psi}{\longrightarrow}\left(R_{c}\right)^{\mathbb{G}_{m}}\left[y^{ \pm 1}\right] \stackrel{y \mapsto 1}{\longrightarrow}\left(R_{c}\right)^{\mathbb{G}_{m}}
$$

is a homomorphism of $\left(R_{c}\right)^{\mathbb{G}_{m}}$-algebras with $\operatorname{ker}(\pi)=(s-1) R_{c}$. In particular, $\pi$ is surjective. For $a \in R_{c}, \pi(a)$ is given by substituting $t=\sqrt[d]{s}$ in $\varphi(a)$, which makes sense because $\varphi(a) \in R_{c}\left[t^{ \pm d}\right]$.

(c) The composition

$$
R_{c} \stackrel{\varphi}{\longrightarrow} R_{c}\left[t^{ \pm 1}\right] \stackrel{\pi}{\longrightarrow}\left(R_{c}\right)^{\mathbb{G}_{m}}\left[t^{ \pm 1}\right]
$$

(with $\pi$ applied coefficient-wise) is injective and makes $\left(R_{c}\right)^{\mathbb{G}_{m}}\left[t^{ \pm 1}\right]$ into an $R_{c}$-module that is generated by d elements.

(d) Let $A$ be a ring with a homomorphism $\left(R_{c}\right)^{\mathbb{G}_{m}} \rightarrow A$. Then

$$
\left(A \otimes_{\left(R_{c}\right)^{\mathbb{G}_{m}}} R\right)^{\mathbb{G}_{m}}=A .
$$

Proof. (a) To show injectivity, let $f \in\left(R_{c}\right)^{\mathbb{G}_{m}}\left[y^{ \pm 1}\right]$ with $f(s)=0$. Then

$$
0=\varphi(f(s))=f(\varphi(s))=f\left(s t^{-d}\right),
$$

so $f=0$. For surjectivity, let $a \in R_{c}$ and first assume $a$ to be a semi-invariant of weight $k$ with $k \in \mathbb{Z}$. Obtain $k=q d+r$ with $q, r \in \mathbb{Z}, 0 \leqslant r<d$ by division with 
remainder. It follows that $s^{q} a$ is a semi-invariant of degree $r$ and therefore an invariant, so $a=s^{q} a \cdot s^{-q} \in\left(R_{c}\right)^{\mathbb{G}_{m}}\left[s^{ \pm 1}\right]$. We also obtain $r=0$, so $k$ is divisible by $d$. For $a \in R_{c}$ arbitrary write $\varphi(a)=\sum_{k} a_{k} t^{k}$. Since $\varphi$ defines a $\mathbb{G}_{m}$-action, it follows that each $a_{k}$ is a semi-invariant of weight $k$ and that $a=\sum_{k} a_{k}$. Since all $a_{k}$ lie in $\left(R_{c}\right)^{\mathbb{G}_{m}}\left[s^{ \pm 1}\right]$, surjectivity follows.

(b) The first claim is clear. Regarding the second claim, we have shown above that $\varphi(a) \in$ $R_{c}\left[t^{ \pm d}\right]$ for $a \in R_{c}$. For showing that the map that is claimed to be equal to $\pi$ really is $\pi$, it suffices to check this for $s$, which is straightforward.

(c) Let $a \in R_{c}$. By (a) we have $a=f(s)$ with $f \in\left(R_{c}\right)^{\mathbb{G}_{m}}\left[y^{ \pm 1}\right]$. So

$$
\pi(\varphi(a))=\pi(f(\varphi(s)))=\pi\left(f\left(s t^{-d}\right)\right)=f\left(\pi(s) t^{-d}\right)=f\left(t^{-d}\right)
$$

from which (c) follows.

(d) By (a), we have $R^{\prime}:=A \otimes_{\left(R_{c}\right)^{\mathbb{G}_{m}}} R=A\left[(1 \otimes s)^{ \pm 1}\right]$. By definition, $\left(R^{\prime}\right)^{\mathbb{G}_{m}}=\operatorname{ker}\left(\varphi^{\prime}-\mathrm{id}\right)$ with $\varphi^{\prime}: R^{\prime} \rightarrow R^{\prime}\left[t^{ \pm 1}\right]$ obtained by tensoring $\varphi$. Let $r \in R^{\prime}$ and write $r=\sum_{i \in \mathbb{Z}} a_{i}(1 \otimes s)^{i}$ with $a_{i} \in A$. Then

$$
\varphi^{\prime}(r)-r=\sum_{i} a_{i}\left(\left(1 \otimes t^{-d} s\right)^{i}-(1 \otimes s)^{i}\right)=\sum_{i} a_{i}(1 \otimes s)^{i}\left(t^{-i d}-1\right),
$$

which is zero if and only if $a_{i}=0$ for $i \neq 0$, i.e., $r \in A$.

Again we present a simple example to illustrate the theorem.

Example 3.2. Consider the action of $\mathbb{G}_{m}$ on $X=\mathbb{C}^{2}$ by $\mathbb{C}^{\times} \ni \beta \mapsto\left(\begin{array}{cc}\beta^{2} & 0 \\ 0 & \beta^{3}\end{array}\right)$. The action is given by

$$
\varphi: R=\mathbb{C}\left[a_{1}, a_{2}\right] \rightarrow \mathbb{C}\left[a_{1}, a_{2}\right]\left[t^{ \pm 1}\right], a_{1} \mapsto t^{2} a_{1}, a_{2} \mapsto t^{3} a_{2} .
$$

So $s=a_{1} / a_{2}$ is a local slice of degree 2 with denominator $c=a_{1} a_{2}$. (Recall that denominator is a technical term here, given by the definition of a local slice.) By the last statement of Theorem 3.1(b), $\pi: R_{c}=\mathbb{C}\left[a_{1}^{ \pm 1}, a_{2}^{ \pm 1}\right] \rightarrow\left(R_{c}\right)^{\mathbb{G}_{m}}$ is given by $\pi\left(a_{1}\right)=s^{2} a_{1}=a_{1}^{3} / a_{2}^{2}$ and $\pi\left(a_{2}\right)=$ $s^{3} a_{2}=a_{1}^{3} / a_{2}^{2}$, which yields the invariant ring

$$
\mathbb{C}\left[a_{1}^{ \pm 1}, a_{2}^{ \pm 1}\right]^{\mathbb{G}_{m}}=\mathbb{C}\left[\frac{a_{1}^{3}}{a_{2}^{2}}, \frac{a_{2}^{2}}{a_{1}^{3}}\right] .
$$

Similarly to Example 2.2, the quotient $\mathbb{C}^{\times} \times \mathbb{C}^{\times} \rightarrow \mathbb{C}^{\times}$given by the invariant ring is excellent, and it is elementary to check that the fibers are $\mathbb{G}_{m}$-orbits.

It is also easy to see that $\mathbb{C}\left[a_{1}, a_{2}^{ \pm 1}\right]^{\mathbb{G}_{m}}=\mathbb{C}\left[a_{1}^{3} / a_{2}^{2}\right]$. The corresponding quotient $\mathbb{C} \times \mathbb{C}^{\times} \rightarrow \mathbb{C}$ is interesting for the following reason: Every $\mathbb{G}_{m}$-orbit is isomorphic to $\mathbb{G}_{m}$. Having the same dimension, all orbits are closed, so by Mumford et al. [17, Amplification 1.3] the quotient is universally geometric. However, the quotient has no cross section. Indeed, a cross section would correspond to a homomorphism $\pi: \mathbb{C}\left[a_{1}, a_{2}^{ \pm 1}\right] \rightarrow \mathbb{C}\left[a_{1}^{3} / a_{2}^{2}\right]$ fixing $\mathbb{C}\left[a_{1}^{3} / a_{2}^{2}\right]$. Since $a_{2}$ is invertible, it would be mapped to some $\gamma \in \mathbb{C}^{\times}$, and then

$$
\pi\left(a_{1}\right)^{3}=\frac{\pi\left(a_{1}\right)^{3} \gamma^{2}}{\pi\left(a_{2}\right)^{2}}=\gamma^{2} \pi\left(\frac{a_{1}^{3}}{a_{2}^{2}}\right)=\frac{\gamma^{2} a_{1}^{3}}{a_{2}^{2}},
$$

which is impossible since $a_{1}^{3} / a_{2}^{2}$ has no third root in $\mathbb{C}\left[a_{1}^{3} / a_{2}^{2}\right]$. This shows that an excellent quotient is stronger than a universally geometric quotient with isomorphic fibers.

\section{Solvable Group aCtions}

In this section $G$ is a connected solvable linear algebraic group over an algebraically closed field $K$, unless stated otherwise. Let us summarize the relevant facts about its structure (see Humphreys [15, Section 19] and Rosenlicht [20, Corollary 2, page 101])): As a variety, we may write $G$ as

$$
G=\left\{\left(\xi_{1}, \ldots, \xi_{l}, \eta_{1}, \ldots, \eta_{m}\right) \in K^{l+m} \mid \eta_{1} \cdots \eta_{m} \neq 0\right\}
$$

such that 
(1) for $i=1, \ldots, l$, the subvariety

$$
G_{i}:=\left\{\left(\xi_{1}, \ldots, \xi_{i}, 0, \ldots, 0,1, \ldots, 1\right)\right\} \subseteq G
$$

is a normal subgroup;

(2) the map $G_{i} \rightarrow \mathbb{G}_{a},\left(\xi_{1}, \ldots, \xi_{i}, 0, \ldots, 0,1, \ldots, 1\right) \mapsto \xi_{i}$ is a morphism of algebraic groups;

(3) with $T$ the $m$-dimensional torus, the map $G \rightarrow T,\left(\xi_{1}, \ldots, \xi_{l}, \eta_{1}, \ldots, \eta_{m}\right) \mapsto\left(\eta_{1}, \ldots, \eta_{m}\right)$ is a morphism of algebraic groups.

It follows that the conjugation action of $G$ on each $G_{i} / G_{i-1} \cong \mathbb{G}_{a}$ is given by a character $\chi_{i} \in K[G]$. If $K[G]=K\left[z_{1}, \ldots, z_{l}, t_{1}^{ \pm 1}, \ldots, t_{m}^{ \pm 1}\right]$ then the $\chi_{i}$ are power products of the $t_{j}^{ \pm 1}$.

Let $G$ act on an irreducible affine variety $X$. We present an algorithm for producing a local slice $s \in R:=K[X]$ for the action of the subgroup $G_{1} \cong \mathbb{G}_{a}$ such that the denominator $c$ is a semi-invariant. We assume that it is possible to perform addition, multiplication, and zero testing of elements of $R$. Notice that the algorithm does not require any Gröbner basis computations and not even linear algebra (unless the underlying computations in $R$ require Gröbner bases).

Algorithm 4.1 (A local slice for the additive group with semi-invariant denominator).

Input: An action of a connected solvable group $G$ on an irreducible affine variety $X$, given by a homomorphism

$$
\varphi: R \rightarrow R\left[z_{1}, \ldots, z_{l}, t_{1}^{ \pm 1}, \ldots, t_{m}^{ \pm 1}\right],
$$

where $K[X]=R$ and $K[G]=K\left[z_{1}, \ldots, z_{l}, t_{1}^{ \pm 1}, \ldots, t_{m}^{ \pm 1}\right]$. Assume that the character $\chi_{1} \in K\left[t_{1}^{ \pm 1}, \ldots, t_{m}^{ \pm 1}\right]$ as above is given, and that the subgroup $G_{1} \cong \mathbb{G}_{a}$ acts nontrivially.

Output: A local slice $s \in R$ with denominator $c$ for the action of $G_{1}$ such that $c$ is a semi-invariant of $G$.

(1) Compute a local slice $s_{1} \in R$ for the action of the subgroup $G_{1}$ with denominator $c_{1} \in$ $R^{G_{1}}$, using the algorithm of Sancho de Salas [22, Section 3] or Tanimoto [24, Section 3].

(2) For $i=2, \ldots, l$ repeat step 3.

(3) With $\varphi_{i}: R \rightarrow R\left[z_{1}, \ldots, z_{i}\right]$ corresponding to the action of $G_{i}$, compute $\varphi_{i}\left(c_{i-1}\right)$ (which, as we will see, is nonzero and lies in $R\left[z_{i}\right]$ ) and let $k$ be its degree. Set $c_{i}$ and $s_{i}$ to be the coefficient of $z_{i}^{k}$ in $\varphi_{i}\left(c_{i-1}\right)$ and $\varphi_{i}\left(s_{i-1}\right)$, respectively. We will show that $s_{i}$ is a local slice for the action of $G_{1}$ with denominator $c_{i} \in R^{G_{i}}$.

(4) Compute $\varphi\left(c_{l}\right) \in R\left[t_{1}^{ \pm 1}, \ldots, t_{m}^{ \pm 1}\right]$ and choose a power product $t^{*}$ of the $t_{j}^{ \pm 1}$ occurring in it. Since there is some freedom in the choice of $t^{*}$, this can be used to make the coefficient of $t^{*}$ in $\varphi\left(c_{l}\right)$ as palatable as possible. For example, a constant from $K$ would be very desirable. With $d$ the degree of $s_{1}$, define $s$ to be the coefficient of $t^{*} \cdot \chi_{1}^{d}$ in $\varphi\left(s_{l}\right)$, and $c$ to be the coefficient of $t^{*}$ in $\varphi\left(c_{l}\right)$. We will show that $s$ is a local slice with denominator $c$, and $c$ is a semi-invariant.

Proof of correctness of Algorithm 4.1. For $i=1, \ldots, l$, we claim that $s_{i}$ is a local slice of degree $d$ with denominator $c_{i} \in R^{G_{i}}$. This is true for $i=1$, so let us assume $i \geqslant 2$. By induction, $0 \neq c_{i-1} \in R^{G_{i-1}}$. Since $\varphi_{i}$ defines a group action, $\varphi_{i}\left(c_{i-1}\right) \neq 0$. More precisely, the action of $G_{i} / G_{i-1} \cong \mathbb{G}_{a}$ on $R^{G_{i-1}}$ is given by $\varphi_{i}: R^{G_{i-1}} \rightarrow R^{G_{i-1}}\left[z_{i}\right]$. With (2.1) applied to $\varphi_{i}\left(c_{i-1}\right)$, this shows that $c_{i}$, defined in step 3 , lies in $R^{G_{i}}$. Write $\varphi_{1}\left(s_{i-1}\right)=\sum_{j=0}^{d} r_{j} z_{1}^{j}$ with $r_{j} \in R$, so $r_{d}=c_{i-1}$. Applying Lemma 4.2, which is proved below, to $G_{1} \subseteq G_{i}$ yields

$$
\left(\operatorname{id}_{K\left[z_{1}, \ldots, z_{i}\right]} \otimes \varphi_{1}\right)\left(\varphi_{i}\left(s_{i-1}\right)\right)=\sum_{j=0}^{d} \varphi_{i}\left(r_{j}\right) z_{1}^{j},
$$

since the conjugation action of $G_{i}$ on $G_{1}$ is trivial. Comparing the coefficients of $z_{1}^{j} z_{i}^{k}$ in this equation shows that $\varphi_{1}\left(s_{i}\right)$, with $s_{i}$ defined in step 3 , equals a polynomial of degree $d$ in $R\left[z_{1}\right]$ with highest coefficient $c_{i}$. This proves our claim.

We now look at step 4 . The torus $T$ acts on $R^{G_{l}}$, to which $c_{l}$ belongs. As for actions of the multiplicative group, it follows that each coefficient of $\varphi\left(c_{l}\right)$ is a semi-invariant, so this is true 
for $c$. As above, Lemma 4.2 yields

$$
\left(\operatorname{id}_{K\left[z_{1}, \ldots, z_{l}, t_{1}^{ \pm 1}, \ldots, t_{m}^{ \pm 1}\right]} \otimes \varphi_{1}\right)\left(\varphi\left(s_{l}\right)\right)=\sum_{j=0}^{d} \chi_{1}^{j} \varphi\left(r_{j}\right) z_{1}^{j}
$$

with $r_{j} \in R, r_{d}=c_{l}$. Comparing the coefficient of $z_{1}^{j} \cdot t^{*} \cdot \chi_{1}^{d}$ shows that $\varphi_{1}(s)$ is a polynomial of degree $d$ with highest coefficient $c$.

The following lemma was used in the proof:

Lemma 4.2. Let $G$ be a linear algebraic group acting on an affine variety $X$, and let $H \subseteq G$ be a normal subgroup with $H \cong \mathbb{G}_{a}$. With $R=K[X]$ and $\varphi_{G}: R \rightarrow K[G] \otimes R, \varphi_{H}: R \rightarrow R[z]$ corresponding to the actions of $G$ and $H$, let $s \in R$ and write $\varphi_{H}(s)=\sum_{i=0}^{d} r_{i} z^{i}$. Then

$$
\left(\operatorname{id}_{K[G]} \otimes \varphi_{H}\right)\left(\varphi_{G}(s)\right)=\sum_{i=0}^{d} \chi^{i} \varphi_{G}\left(r_{i}\right) z^{i}
$$

where the character $\chi \in K[G]$ corresponds to the conjugation action of $G$ on $H$.

Proof. The commutative diagram

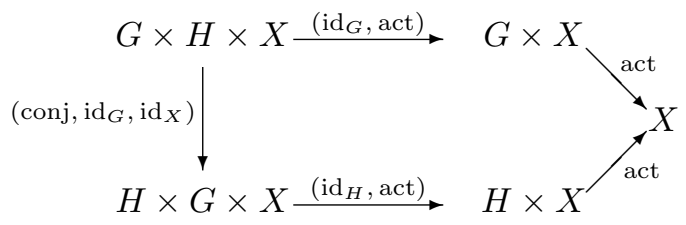

gives rise to a commutative diagram of rings, from which the lemma follows. Observe that the conjugation action $G \times H \rightarrow H$ corresponds to the map $K[z] \rightarrow K[G][z]$ sending $z$ to $\chi \cdot z$.

We will also need an algorithm that finds a local slice $s$ for an action of a multiplicative group such that its denominator $c$ is a semi-invariant with respect to the action of an ambient torus. So let $T$ be an $m$-dimensional torus acting on an affine variety $X$. With $K[X]=: R$, assume that the action is given by a homomorphism $\varphi: R \rightarrow R\left[t_{1}^{ \pm 1}, \ldots, t_{m}^{ \pm 1}\right]$. As in Section 3 , for a semi-invariant $a \in R$ with $\varphi(a)=t_{1}^{e_{1}} \cdots t_{m}^{e_{m}} a$, the weight is $w(a):=e_{1}$.

Algorithm 4.3 (A local slice for the multiplicative group with a semi-invariant denominator).

Input: A torus action on an irreducible affine variety given by a homomorphism $\varphi$ as above. Assume that generators $a_{1}, \ldots, a_{n}$ of the $K$-algebra $R$ are given, and that the first direct component $T_{1} \cong \mathbb{G}_{m}$ of $T$ acts nontrivially.

Output: A local slice $s \in R_{c}$ with denominator $c$ for the action of $T_{1}$, such that $s$ and $c$ are semi-invariants of $T$.

(1) Collect all coefficients of the $\varphi\left(a_{i}\right)$ into a set $\left\{b_{1}, \ldots, b_{l}\right\}$. Thus the $b_{i}$ are semi-invariants.

(2) Let $d$ be the gcd of the weights $w\left(b_{i}\right)$ and find integers $k_{1}, \ldots, k_{l}$ such that $d=\sum_{i=1}^{l} k_{i} w\left(b_{i}\right)$.

(3) Set $c$ to be the product of all $b_{i}$ with $k_{i} \neq 0$ and $s:=\prod_{i=1}^{l} b_{i}^{-k_{i}}$.

Proof of correctness of Algorithm 4.3. It is clear that $c$ is a semi-invariant and that $s$ is invertible of $R_{c}$ and a semi-invariant of weight $-d$. Since $\varphi\left(a_{i}\right) \in R_{c}\left[t_{1}^{ \pm d}, t_{2}^{ \pm 1}, \ldots, t_{m}^{ \pm 1}\right]$ for all $i$, the image $\varphi\left(R_{c}\right)$ lies in that ring. Therefore an element of $R_{c} \backslash R_{c}^{T_{1}}$ has degree at least $d$.

We are now ready to present the centerpiece of this paper: an algorithm that computes the invariant ring $\left(K[X]_{c}\right)^{G}$ of a connected solvable group, with $c$ a semi-invariant. This yields an excellent quotient of $X_{c}$ (see Theorem 4.7(b)). The algorithm has been implemented in the computer algebra system MAGMA [2], but the implementation is limited to the case that $R$ is a polynomial ring over a field of characteristic 0 .

Algorithm 4.4 (Solvable group invariants). 
Input: - The coordinate $\operatorname{ring} K[G]=K\left[z_{1}, \ldots, z_{l}, t_{1}^{ \pm 1}, \ldots, t_{m}^{ \pm 1}\right]$ of a connected solvable group, and the characters $\chi_{i} \in K\left[t_{1}^{ \pm 1}, \ldots, t_{m}^{ \pm 1}\right]$ defining the conjugation action on $G_{i} / G_{i-1}$ (see at the beginning of this section).

- The coordinate $\operatorname{ring} R:=K[X]=K\left[a_{1}, \ldots, a_{n}\right]$ of an irreducible affine variety. Assume that addition, multiplication and zero recognition can be carried out in $R$.

- An action of $G$ on $X$, given by a homomorphism $\varphi: R \rightarrow R\left[z_{1}, \ldots, z_{l}, t_{1}^{ \pm 1}, \ldots, t_{m}^{ \pm 1}\right]$.

Output: - A nonzero semi-invariant $c \in R$.

- Invariants $b_{0}, \ldots, b_{n} \in R_{c}$ such that

$$
\left(R_{c}\right)^{G}=K\left[b_{0}, b_{1}, \ldots, b_{n}\right] .
$$

Moreover, the homomorphism

$$
\pi: R_{c} \rightarrow\left(R_{c}\right)^{G}, c^{-1} \mapsto b_{0}, a_{j} \mapsto b_{j} \quad(j=1, \ldots, n)
$$

satisfies $\pi^{2}=\pi$, so it is a projection onto the invariant ring.

- Generators $u_{1}, \ldots, u_{k} \in R$ of $\operatorname{ker}(\pi)$, where

$$
k=\operatorname{dim}\left(R_{c}\right)-\operatorname{dim}\left(\left(R_{c}\right)^{G}\right) .
$$

(1) Initialization: Set $b_{0}:=c:=1, b_{j}:=a_{j}(j=1, \ldots, n)$, and $k:=0$.

(2) For $i=1, \ldots, l$ repeat steps $3-7$.

(3) If none of the $\varphi\left(b_{j}\right)(j=0, \ldots, n)$ involves $z_{i}$, skip steps $4-7$ and proceed with the next $i$.

(4) Apply Algorithm 4.1 with the following arguments: the ring $\widetilde{R}:=K\left[b_{0}, \ldots, b_{n}\right] \subseteq R_{c}$, the homomorphism $\widetilde{R} \rightarrow \widetilde{R}\left[z_{i}, \ldots, z_{l}, t_{1}^{ \pm 1}, \ldots, t_{m}^{ \pm 1}\right]$ obtained by extending $\varphi$ to $R_{c}$ and restricting to $\widetilde{R}$, and the character $\chi_{i}$. Let $\widetilde{s} \in \widetilde{R}$ be the resulting local slice with denominator $\widetilde{c}$.

(5) With $\varphi_{i}: \widetilde{R} \rightarrow \widetilde{R}\left[z_{i}\right]$ corresponding to the action of the subgroup $G_{i}$, perform division with remainder in $\widetilde{R}_{\widetilde{c}}\left[z_{i}\right]$ :

$$
\varphi_{i}\left(b_{j}\right)=g_{j} \cdot \varphi_{i}(\widetilde{s})+r_{j} \quad(j=1, \ldots, n) .
$$

Then $r_{j} \in\left(\widetilde{R}_{\widetilde{c}}\right)^{G_{i}}$.

(6) Choose $e$ large enough such that $c^{e} \widetilde{c} \in R$ and set $c^{\prime}:=c^{e+1} \widetilde{c}, r_{0}:=\left(c^{\prime}\right)^{-1} \in R_{c^{\prime}}$. Choose $\hat{e}$ large enough such that $u_{k+1}:=c^{\hat{e}} \widetilde{s} \in R$.

(7) Replace $b_{j}$ by $r_{j}(j=0, \ldots, n), c$ by $c^{\prime}$, and $k$ by $k+1$.

(8) For $i=1, \ldots, m$ repeat steps $9-13$.

(9) If none of the $\varphi\left(b_{j}\right)(j=0, \ldots, n)$ involves $t_{i}$, skip steps 10-13 and proceed with the next $i$.

(10) Apply Algorithm 4.3 to the ring $\widetilde{R}:=K\left[b_{0}, \ldots, b_{n}\right] \subseteq R_{c}$ and the homomorphism $\widetilde{R} \rightarrow \widetilde{R}\left[t_{i}^{ \pm 1}, \ldots, t_{m}^{ \pm 1}\right]$ obtained by extending $\varphi$ to $R_{c}$ and restricting to $\widetilde{R}$. Let $\widetilde{s} \in \widetilde{R}_{\widetilde{c}}$ be the resulting local slice of degree $d$ with denominator $\widetilde{c} \in \widetilde{R}$.

(11) With $\psi_{i}: \widetilde{R} \rightarrow \widetilde{R}\left[t_{i}^{ \pm d}\right]$ corresponding to the action of the subgroup

$$
T_{i}:=\left\{\left(\xi_{1}, \ldots, \xi_{l}, \eta_{1}, \ldots, \eta_{i}, 1, \ldots, 1\right) \in K^{l+m} \mid \eta_{1} \cdots \eta_{i} \neq 0\right\} \subseteq G,
$$

obtain $r_{j} \in\left(\widetilde{R}_{\widetilde{c}}\right)^{T_{i}}$ by substituting $t_{i}=\sqrt[d]{\widetilde{s}}$ in $\psi_{i}\left(b_{j}\right)(j=1, \ldots, n)$.

(12) Choose $e$ large enough such that $c^{e} \widetilde{c} \in R$ and set $c^{\prime}:=c^{e+1} \widetilde{c}$. Obtain $r_{0} \in \widetilde{R}_{\widetilde{c}}=R_{c^{\prime}}$ by substituting $t_{i}=\sqrt[d]{\widetilde{s}}$ in $\psi_{i}\left(b_{0}^{e+1} \widetilde{c}^{-1}\right)$. Choose $\hat{e}$ such that $u_{k+1}:=\left(c^{\prime}\right)^{\hat{e}}(\widetilde{s}-1) \in R$.

(13) Replace $b_{j}$ by $r_{j}(j=0, \ldots, n), c$ by $c^{\prime}$, and $k$ by $k+1$.

Remark 4.5. If the $\operatorname{ring} R$ in Algorithm 4.4 is given as a quotient ring of a polynomial ring, $R=K\left[x_{1}, \ldots, x_{n}\right] / I$, then the algorithm yields the following presentation of $\left(R_{c}\right)^{G}$ : Let $U_{i} \in$ $K\left[x_{1}, \ldots, x_{n}\right]$ be a preimage of $u_{i} \in R(i=1, \ldots, k)$ and let $U_{0}$ be a preimage of $c$. Then it is easy to see that the kernel of the map

$$
K\left[x_{0}, \ldots, x_{n}\right] \rightarrow\left(R_{c}\right)^{G}, x_{j} \mapsto b_{j}
$$

is generated by $I, U_{1}, \ldots, U_{k}$, and $x_{0} U_{0}-1$. Thus the algorithm computes the quotient $X_{c} / G$ as an affine variety in $K^{n+1}$. 
Proof of correctness of Algorithm 4.4. Using induction on $i$, we first show that steps 1-7 compute the correct output for the subgroup $G_{i}$, with $1 \leqslant i \leqslant l$. By induction, $\widetilde{R}=\left(R_{c}\right)^{G_{i-1}}$, on which $G_{i} / G_{i-1} \cong \mathbb{G}_{a}$ acts. Step 3 checks if this action is trivial, and if it is not, step 4 computes a local slice with denominator $\widetilde{c} \in \widetilde{R}$, which is a semi-invariant of $G$. By Theorem 2.1(b) there is a homomorphism

$$
\pi_{i}: \widetilde{R}_{\widetilde{c}} \rightarrow\left(\widetilde{R}_{\widetilde{c}}\right)^{G_{i}}
$$

of $\left(\widetilde{R}_{\widetilde{c}}\right)^{G_{i}}$-algebras with kernel $\widetilde{s} \widetilde{R}_{\widetilde{c}}$, and step 5 computes the images $r_{j}=\pi_{i}\left(b_{j}\right)$ for $1 \leqslant j \leqslant n$. By induction, we also have a homomorphism $R_{c} \rightarrow \widetilde{R}$ of $\widetilde{R}$-algebras mapping the $a_{j}$ to the $b_{j}$ with kernel generated (as an ideal) by $u_{1}, \ldots, u_{k}$. This extends to a homomorphism $\left(R_{c}\right)_{\widetilde{c}} \rightarrow \widetilde{R}_{\widetilde{c}}$

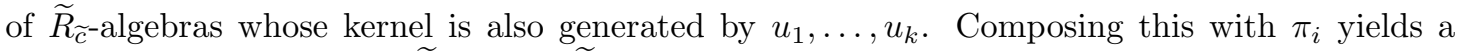
homomorphism $\left(R_{c}\right)_{\widetilde{c}} \rightarrow\left(\widetilde{R}_{\widetilde{c}}\right)^{G_{i}}$ of $\left(\widetilde{R}_{\widetilde{c}}\right)^{G_{i}}$-algebras sending $a_{j}$ to $r_{j}$ with kernel generated by $u_{1}, \ldots, u_{k}, \widetilde{s}$. Step 6 finds a semi-invariant $c^{\prime} \in R$ such that $\left(R_{c}\right)_{\widetilde{c}}=R_{c^{\prime}}$ and chooses $u_{k+1} \in R$ such that $\widetilde{s} R_{c^{\prime}}=u_{k+1} R_{c^{\prime}}$. Moreover, $\left(R_{c^{\prime}}\right)^{G_{i}}=\left(\widetilde{R}_{\widetilde{c}}\right)^{G_{i}}$, so

$$
\begin{aligned}
& \operatorname{dim}\left(R_{c^{\prime}}\right)-\operatorname{dim}\left(\left(R_{c^{\prime}}\right)^{G_{i}}\right)=\operatorname{dim}\left(\left(R_{c}\right) \widetilde{c}\right)-\operatorname{dim}\left(\widetilde{R}_{\widetilde{c}}\right)+\operatorname{dim}\left(\widetilde{R}_{\widetilde{c}}\right)-\operatorname{dim}\left(\left(\widetilde{R}_{\widetilde{c}}\right)^{G_{i}}\right)= \\
&\left(\operatorname{dim}\left(R_{c}\right)-\operatorname{dim}\left(\left(R_{c}\right)^{G_{i-1}}\right)\right)+\left(\operatorname{dim}\left(\widetilde{R}_{\widetilde{c}}\right)-\operatorname{dim}\left(\left(\widetilde{R}_{\widetilde{c}}\right)^{G_{i}}\right)\right)=k+1,
\end{aligned}
$$

where the last equality follows by induction and from Theorem 2.1(a). So indeed after step 7, the current $c, b_{j}$, and $u_{1}, \ldots, u_{k}$ satisfy all the specifications of the algorithm for the group $G_{i}$.

We now turn our attention to steps 8-13 and show that they compute the correct output for the subgroups $T_{i}(1 \leqslant i \leqslant m)$. The proof is almost identical to the one for steps $1-7$, using Theorem 3.1 instead of 2.1. Here the kernel of the projection $\pi_{i}: \widetilde{R}_{\widetilde{c}} \rightarrow\left(\widetilde{R}_{\widetilde{c}}\right)^{T_{i}}$ is $(\widetilde{s}-1) \widetilde{R}_{\widetilde{c}}$. The only other difference is the computation of $r_{0}$ in step 12. For the algorithm to be correct, $r_{0}$ needs to be the image of $\left(c^{\prime}\right)^{-1}$ under the composition $R_{c^{\prime}} \rightarrow \widetilde{R}_{\widetilde{c}} \stackrel{\pi_{i}}{\longrightarrow}\left(\widetilde{R}_{\widetilde{c}}\right)^{T_{i}}$. By induction, the first map sends $c^{-1}$ to $b_{0}$, and it sends $\widetilde{c}$ to itself since $\widetilde{c} \in \widetilde{R}$. So the composition $R_{c^{\prime}} \rightarrow\left(\widetilde{R}_{\widetilde{c}}\right)^{T_{i}}$ sends $\left(c^{\prime}\right)^{-1}$ to $\pi_{i}\left(b_{0}^{e+1} \widetilde{c}^{-1}\right)$, which is precisely what step 12 computes.

The following simple example, which is a follow-up to Example 2.2, should illustrate Algorithm 4.4.

Example 4.6. Let $G \subset \mathrm{SL}_{2}(\mathbb{C})$ be the subgroup consisting of all upper triangular matrices, which is a Borel subgroup. $G$ acts on binary forms of degree 2 by the matrices $\left(\begin{array}{ccc}\beta^{2} & \alpha \beta & \alpha^{2} \\ 0 & 1 & 2 \alpha \beta^{-1} \\ 0 & 0 & \beta^{-2}\end{array}\right)$ with $\alpha, \beta \in \mathbb{C}, \beta \neq 0$. The action is given by $\varphi: R:=\mathbb{C}\left[a_{1}, a_{2}, a_{3}\right] \rightarrow \mathbb{C}\left[a_{1}, a_{2}, a_{3}\right]\left[z, t^{ \pm 1}\right]$ with

$$
\varphi\left(a_{1}\right)=t^{2} a_{1}+z t a_{2}+z^{2} a_{3}, \varphi\left(a_{2}\right)=a_{2}+2 z t^{-1} a_{3}, \varphi\left(a_{3}\right)=t^{-2} a_{3},
$$

extending (2.4). To run Algorithm 4.4, we first need to choose a local slice for $\mathbb{G}_{a} \subset G$, given by $t=1$. By Example 2.2, $s=a_{2}$ is an obvious choice. Luckily, the denominator $c=a_{3}$ already is a semi-invariant. (In fact, running Algorithm 4.1 would leave the initial choice $s=a_{2}$ unchanged, since the power product $t^{*}$ in step 4 would be $t^{*}=t^{-2}$, while the conjugation in $G$ is given by $\chi=t^{2}$.) According to (2.5) in Example 2.2, the first half of Algorithm 4.4 yields the three generators

$$
b_{0}=a_{3}^{-1}, b_{1}=\frac{4 a_{1} a_{3}-a_{2}^{2}}{4 a_{3}}, \text { and } b_{3}=a_{3}
$$

of $\widetilde{R}=R_{c}^{\mathbb{G}_{a}}=\mathbb{C}\left[a_{1}, a_{2}, a_{3}^{ \pm 1}\right]^{\mathbb{G}_{a}}$. By an easy computation, we have

$$
\varphi\left(b_{0}\right)=t^{2} b_{0}, \varphi\left(b_{1}\right)=t^{2} b_{1}, \varphi\left(b_{3}\right)=t^{-2} b_{3} .
$$

So, as predicted by the theory, only the multiplicative group $\mathbb{G}_{m}$ acts on $\widetilde{R}$, and we find a local slice $\widetilde{s}=b_{3}=a_{3}$ of degree 2 for this action. Since $\widetilde{s}$ is already invertible in $\widetilde{R}$, the denominator can be taken to be $\widetilde{c}=1$. From this, steps 11 and 12 of Algorithm 4.4 compute generators $r_{0}=\widetilde{s} b_{0}=1, r_{1}=\widetilde{s} b_{1}=a_{1} a_{3}-a_{2}^{2} / 4$, and $r_{3}=\widetilde{s}^{-1} b_{3}=1$ of $\widetilde{R}^{\mathbb{G}_{m}}=\mathbb{C}\left[a_{1}, a_{2}, a_{3}^{ \pm 1}\right]^{G}$. So

$$
\mathbb{C}\left[a_{1}, a_{2}, a_{3}^{ \pm 1}\right]^{G}=\widetilde{R}^{\mathbb{G}_{m}}=\mathbb{C}\left[r_{0}, r_{1}, r_{2}\right]=\mathbb{C}\left[a_{2}^{2}-4 a_{1} a_{3}\right] .
$$


This is the final result of Algorithm 4.4, together with the projection

$$
\pi: \mathbb{C}\left[a_{1}, a_{2}, a_{3}^{ \pm 1}\right] \rightarrow \mathbb{C}\left[a_{1}, a_{2}, a_{3}^{ \pm 1}\right]^{G}, a_{1} \mapsto a_{1} a_{3}-a_{2}^{2} / 4, a_{2} \mapsto 0, a_{3}^{ \pm 1} \mapsto 1
$$

Part (b) of the following theorem says that $\left(R_{c}\right)^{G}$ affords an excellent quotient. Explicitly, the quotient is the map $\mathbb{C}^{2} \times \mathbb{C}^{\times} \rightarrow \mathbb{C}$ given by evaluating the discriminant. It is straightforward to verify that the fibers of this map are indeed $G$-orbits.

It follows directly from (4.2) that the nonlocalized invariant ring is $\mathbb{C}\left[a_{1}, a_{2}, a_{3}\right]^{G}=\mathbb{C}\left[a_{2}^{2}-\right.$ $\left.4 a_{1} a_{3}\right]$. This makes the business about using a localization look like a diversion. However, the corresponding map $\mathbb{C}^{3} \rightarrow \mathbb{C}$ is not a geometric quotient. In fact, a short calculation shows that all fibers, except for the zero fiber, contain precisely three $G$-orbits. But the nonlocalized ring $\mathbb{C}\left[a_{1}, a_{2}, a_{3}\right]$ has the advantage that $\mathrm{SL}_{2}$ acts on it, with $G$ a Borel subgroup. So the result mentioned in the first paragraph of the introduction yields

$$
\mathbb{C}\left[a_{1}, a_{2}, a_{3}\right]^{\mathrm{SL}_{2}}=\mathbb{C}\left[a_{2}^{2}-4 a_{1} a_{3}\right],
$$

so we recover a very well-known result about invariants of binary forms. Notice that this invariant ring was also used in Derksen and Kemper [4, Example 4.1.12] to give an illustrating example of the Derksen algorithm. In that example, a Gröbner basis computation is needed which cannot be shown, whereas here all computations are elementary. However, it was a lucky circumstance in this example that the $G$-invariants of the nonlocalized ring could easily be determined from the $G$-invariants of the localized ring.

The following theorem summarizes the results of this paper:

Theorem 4.7. Let $G$ be a connected solvable linear algebraic group over an algebraically closed field $K$, acting on an irreducible affine variety $X$.

(a) Algorithm 4.4 computes a nonzero semi-invariant $c \in R:=K[X]$, generators of the invariant ring $\left(R_{c}\right)^{G}$, and the relations between the generators. If no Gröbner bases are required for computing in $R$, then the algorithm does not require any Gröbner basis computations either.

(b) With $X_{c} / G$ is the variety corresponding to $\left(R_{c}\right)^{G}$, the map $X_{c} \rightarrow X_{c} / G$ is an excellent quotient with fibers $\mathbb{A}^{r, s}$ as defined in the introduction. If $G$ is unipotent, then $s=0$.

(c) The generators of $\left(R_{c}\right)^{G}$ also generate the invariant field $K(X)^{G}$ as an extension of $K$.

(d) If $R$ is a complete intersection (e.g. if $R$ is a polynomial ring), then so is $\left(R_{c}\right)^{G}$.

Proof. (a) This follows from the proof of correctness of the algorithm, from Remark 4.5, and by verifying that no step in the algorithm requires any Gröbner bases.

(b) We have already seen in the proof of correctness of the algorithm that steps 2-7 perform the passage from $\left(R_{c}\right)^{G_{i-1}}$ to $\left(R_{c^{\prime}}\right)^{G_{i}}$. Now it follows from Theorem 2.1 and the remark preceding it that $\operatorname{Spec}\left(\left(R_{c^{\prime}}\right)^{G_{i-1}}\right) \rightarrow \operatorname{Spec}\left(\left(R_{c^{\prime}}\right)^{G_{i}}\right)$ is an excellent quotient with fibers $\mathbb{A}^{1}$. Likewise, Theorem 3.1 shows that steps 9-13 yield an excellent quotient $\operatorname{Spec}\left(\left(R_{c^{\prime}}\right)^{T_{i-1}}\right) \rightarrow \operatorname{Spec}\left(\left(R_{c^{\prime}}\right)^{T_{i}}\right)$ with fibers $\mathbb{A}^{1} \backslash\{0\}$. Applying Theorem 1.2 repeatedly to the chain of excellent quotients produced by the algorithm, we obtain the assertion (b).

(c) This follows from (b) and the fact that for any geometric quotient $X^{\prime} \rightarrow Y$ with $X^{\prime}$ an integral scheme, $K(Y)=K\left(X^{\prime}\right)^{G}$ (see Kemper [16, Lemma 2.9]).

(d) By hypothesis we have $R=K\left[x_{1}, \ldots, x_{n}\right] / I$ with $I$ generated by $n-\operatorname{dim}(R)$ elements. By Remark 4.5 we have an epimorphism $K\left[x_{0}, \ldots, x_{n}\right] \rightarrow\left(R_{c}\right)^{G}$ whose kernel is generated by $n-\operatorname{dim}(R)+k+1=n+1-\operatorname{dim}(R)+\operatorname{dim}\left(R_{c}\right)-\operatorname{dim}\left(\left(R_{c}\right)^{G}\right)=n+1-\operatorname{dim}\left(\left(R_{c}\right)^{G}\right)$ elements, where (4.1) was used for the second equality. This completes the proof.

Apart from dealing with a more general setting, the preprint [16] contains a small number of examples of actions of connected groups where no nonempty open subset exists that admits an excellent quotient. Moreover, actions where a nonempty open subset admits an excellent quotient with fibers $\mathbb{A}^{r, s}$ are characterized as "essentially solvable." These results prompt us to conclude the paper with the following rather bold conjecture:

Conjecture 4.8. If $G$ is a linear algebraic group such that every irreducible affine $G$-variety $X$ has a nonempty $G$-stable subset $U$ that admits an excellent quotient, then $G$ is connected and solvable. 


\section{REFERENCES}

[1] Gergely Bérczi, Brent Doran, Thomas Hawes, Frances Kirwan, Geometric invariant theory for graded unipotent groups and applications, preprint, available at https://arxiv.org/abs/1601.00340, 2016.

[2] Wieb Bosma, John J. Cannon, Catherine Playoust, The Magma Algebra System I: The User Language, J. Symb. Comput. 24 (1997), 235-265.

[3] Harm Derksen, Gregor Kemper, Computing invariants of algebraic group actions in arbitrary characteristic, Adv. Math. 217 (2008), 2089-2129.

[4] Harm Derksen, Gregor Kemper, Computational Invariant Theory, Encyclopaedia of Mathematical Sciences 130, second edn., Springer, Heidelberg, Heidelberg, New York, Dordrecht, London 2015.

[5] Arno van den Essen, An Algorithm to Compute the Invariant Ring of a $G_{a}$-Action on an Affine Variety, J. Symbolic Comput. 16 (1993), 551-555.

[6] Amassa Fauntleroy, Geometric invariant theory for general algebraic groups, Compositio Math. 55 (1985), $63-87$.

[7] Amassa Fauntleroy, Invariant theory for linear algebraic groups. II. (char $k$ arbitrary), Compositio Math. 68 (1988), 23-29.

[8] Walter Ricardo Ferrer Santos, Alvaro Rittatore, Actions and invariants of algebraic groups, Monographs and Research Notes in Mathematics, second edn., CRC Press, Boca Raton, FL 2017.

[9] Gene Freudenburg, Algebraic theory of locally nilpotent derivations, Encyclopaedia of Mathematical Sciences 136, Springer-Verlag, Berlin 2006.

[10] Ulrich Görtz, Torsten Wedhorn, Algebraic geometry I, Advanced Lectures in Mathematics, Vieweg + Teubner, Wiesbaden 2010.

[11] Gert-Martin Greuel, Gerhard Pfister, Geometric quotients of unipotent group actions, Proc. London Math. Soc. (3) 67 (1993), 75-105.

[12] Gert-Martin Greuel, Gerhard Pfister, Geometric quotients of unipotent group actions II, in: Singularities (Oberwolfach, 1996), pp. 27-36, Birkhäuser, Basel 1998

[13] F. D. Grosshans, The invariants of unipotent radicals of parabolic subgroups, Invent. Math. 73 (1983), 1-9.

[14] G. Hochschild, G. D. Mostow, Unipotent groups in invariant theory, Proc. Nat. Acad. Sci. U.S.A. 70 (1973), 646-648.

[15] James E. Humphreys, Linear Algebraic Groups, Springer-Verlag, Berlin, Heidelberg, New York 1981.

[16] Gregor Kemper, Quotients by Connected Solvable Groups, preprint, available at https://arxiv.org/abs/ 1712.03838, 2017.

[17] David Mumford, John Fogarty, Frances Kirwan, Geometric Invariant Theory, Ergebnisse der Math. und ihrer Grenzgebiete 34, third edn., Springer-Verlag, Berlin, Heidelberg, New York 1994.

[18] Vladimir L. Popov, Birational splitting and algebraic group actions, Eur. J. Math. 2 (2016), 283-290.

[19] Maxwell Rosenlicht, Some basic theorems on algebraic groups, Amer. J. Math. 78 (1956), 401-443.

[20] Maxwell Rosenlicht, Questions of rationality for solvable algebraic groups over nonperfect fields, Ann. Mat. Pura Appl. 61 (1963), 97-120.

[21] Maxwell Rosenlicht, A Remark on Quotient Spaces, Anais Acad. Brasil. Ci. 35 (1963), 487-489.

[22] Carlos Sancho de Salas, Invariant theory for unipotent groups and an algorithm for computing invariants, Proc. London Math. Soc. (3) 81 (2000), 387-404.

[23] Lin Tan, An algorithm for explicit generators of the invariants of the basic $G_{a}$-actions, Comm. Algebra 17 (1989), 565-572.

[24] Ryuji Tanimoto, An algorithm for computing the kernel of a locally finite iterative higher derivation, J. Pure Appl. Algebra 212 (2008), 2284-2297.

Technische Universität München, Zentrum Mathematik - M11, Boltzmannstr. 3, 85748 Garching, GERMANY

Email address: kemper@ma.tum.de 